\title{
Neuron-Specific Enolaza as a Marker of Lesion Cerebral Tissue in Patients with Ischemic Stroke
}

\author{
Stadnik SM and Saiko OV* \\ Military Medical Clinical Center of the Western Region, Ukraine
}

*Corresponding author: Saiko OV, Military Medical Clinical Center of the Western region of Ukraine, st.Lychakivska, 26, Lviv,

79010, Ukraine

\section{ARTICLE INFO}

Received: September 24, 2020

Published:

Citation: Stadnik SM, Saiko OV. NeuronSpecific Enolaza as a Marker of Lesion Cerebral Tissue in Patients with Ischemic Stroke. Biomed J Sci \& Tech Res 31(1)2020. BJSTR. MS.ID.005030.

Keywords: Ischemic Stroke; Chronic Cerebral Ischemia; Neuron-Specific Enolase; Neurological Deficit; Cognitive Disorders

Abbreviations: NSE: Neuron-Specific Enolase; mRS: Modified Rankine Scale; VBB: Vertebrobasilar Basin; CHI: Chronic Cerebral Ischemia; CR: Cognitive Disorders

\section{ABSTRACT}

Objective: A promising area in modern angioneurology is the study of markers of brain tissue damage in the serum of patients in the acute period of ischemic stroke and their comparison with the initial level of neurological deficit and the consequences of stroke. The functions of neuron-specific enolase (NSE) are actively studied as a marker of brain tissue damage. The aim is to investigate the level of NSE in the acute period of ischemic stroke.

Material and Methods: The clinical study included 151 patients (mean age $61.5 \pm$ 8.2 years) who divided into 2 groups: the $1^{\text {st }}$ group consisted of 85 patients with acute ischemic stroke; Group 2 - 66 patients with chronic cerebral ischemia. Plasma HCV levels were determined in all patients. Analyzed the correlation NSE with the severity of neurological deficit NIHSS, functional S ac and c u a scale Bartel, level of disability on a scale Rankine severity of cognitive impairment on MMSE. The relationship between NSE levels and survival of patients with ischemic stroke was determined.

Results: In patients of the 1st group, the level of NCE exceeded the rate of patients in the $2^{\text {nd }}$ group by 3.8 times $(\mathrm{p}<0.001)$. Significant differences in the level of NCE depending on the location of the focus of ischemia - in the basin of the left middle cerebral artery and vertebrobasilar basin $(p=0.04)$. In paragraph atsiyentiv with severe neurological deficit exceeded the level of NSE index patients with medium and light neurological deficit at 1,3 $(p=0,251)$ and $2,3(p=0,007)$ times respectively. A correlation was established between the level of NCE and the severity of neurological deficit ( $r=0.67, p=0.027)$, which indicates a direct relationship between the degree of ischemic injury and neurological disorders. In patients with severe cognitive impairment, the level of HCE exceeded that of patients with moderate and mild cognitive impairment by $1.2(\mathrm{p}=0.444)$ and $1.9(\mathrm{p}=$ 0.037 ) times, respectively. When assessing the relationship between the level of NCE with the consequences of stroke, a tendency to higher $\mathrm{n}$ indicators of NCE in the dead $(\mathrm{p}=0$, 083). The limit value of the level of NSE in the blood of patients with ischemic stroke was determined - $40 \mathrm{ng} / \mathrm{ml}$.

Conclusion: Determination of HCE in blood plasma confirms the degree of neuronal damage and is an informative indicator of the presence of neurological deficits in patients with acute ischemic stroke.

Results: The results indicate a potential role for the determination of NCE content in improving the stratification of the risk of death in patients with ischemic stroke.

\section{Introduction}

Stroke - is a medical and social problem, the prevalence of which acquires the features of a global epidemic that threatens the lives and healths population [1]. Deaths from disease of the brain in the structure of total mortality in Ukraine is the second of istse. About $25 \%$ of stroke patients die within the first day Mr. donkey of the disease, $60 \%$ of patients are profound disabilities, and only $n$ ' 
Fifth of returning to work [2-5].With the development of laboratory diagnostics actively in yvchayutsyapathophysiological and marker and prognosis tion stroke, but according to the literature has a low diagnostic kg innist many parameters [6-9]. In recent decades, great attention and chapel yayetsya indicator of brain damage - neuronspecific first enolaz and (NSE) $[10,11]$. Along with this, there is little data on the value of NCE in cerebrovascular diseases.The study of the level of NCE was carried out in various pathologies. Plasma NSA concentrations increase in stroke, Alzheimer's disease, epilepsy, traumatic brain injury, and multiple sclerosis. If the damage neurons NSE enters the blood through the blood-brain bar 'Jer.

In the acute period of stroke there is an immune adjustment, in particular, antibodies to NSE are produced [12].There is an assumption that the level of NSE can correlate and with the severity of brain damage and, thus , the clinical status of patients. However, data on this issue remain contradictory in the literature. Thus, a study by Pandey A, et al. found an increase in NCE in patients with stroke compared with the control group. Index NSE correlated and the level of glucose in patients with hyperglycemia was associated with worse stroke [13]. There was a higher level of NCE in deceased patients. In other studies [14], these changes did not depend on the presence or absence of diabetes. Some authors consider a NSE and independent markers of prognosis of acute ischemic stroke. In other studies, low levels of NSE associated with less about ' in volume infarct [15]. At the same time,Jung CS, et al. [16], having studied the markers of brain damage in the serum of patients with subarachnoid hemorrhage and secondary ischemic brain damage as a result of cerebral vasospasm, concluded that NCE does not correlate with either vasospasm or cerebral ischemia.

Alatas ÖD, et al. [17] identified in patients with netravmatych their internal rishnomozkovymy bleeding you soup biomarker concentrations compared to the control group, but the correlation with NSE about ' in volume hemorrhage in is not found.Dementia is in $\mathrm{OV}$ and sang. Based on previous results last captured or conclude that the NSE level makes it impossible to predict the volume of cerebral ischemic foci after systemic thrombolysis [18]. In the study of NSE in liquor on average 8.5 hours after the onset of clinical symptoms of $\mathrm{R}$ Brouns, et al. detects and $\mathrm{L}$ and differences NSE levels between patients with stroke and control groups [19]. According to the results of other studies, the level of NCE rarely exceeded the norm [20]. At the same time there is evidence that the average level of NSE in serum of patients with ischemic stroke significantly HI th $(p<0,001)$, than in the control group [21].

In other Shih studies NSE index was not associated with stroke etiology of the system TOAST, and not correlated with NIHSS indicator of onset of the disease and about ' in volume of ischemic foci [19].I studied the prognostic value of NSE in patients with stroke. There was an increase in the level of NSE in ischemic stroke in patients with severe motor deficiency, regardless of age, which allowed us to consider this marker as an independent predictor of the consequences of ischemic stroke.At the same time, the NSE index remained stable or decreased until discharge from hospital (compared at with the original index) in patients younger and older, are discharged with clinical improvement [22]. In the works of some authors marked and significant correlation with NSE Rankin scale and that allowed to discuss potential prognostic NSE. Performed at Xia studies to assess functional outcome of 30-day stroke using a modified Rankine scale (mRS) [23].

In idznacheno the level of NSE in the $1^{\text {st }}$ day of illness was significantly correlated with the level of $\mathrm{mRS}$ at 30 days $(r=0,744$, $p<0.001$ ), the average concentration of NSE in patients with $m R S \geq 3$ (worst result) was significantly $\mathrm{HI}$ th compared with patients with $\mathrm{mRS}<3$ (best result) ( $\mathrm{p}<0,001)$. Similar results were obtained in other studies [24] that assessed functional neurological deficits by NIHSS: found a significant correlation between HCE and N IHSS levels on day $60(\mathrm{r}=0.461, \mathrm{p}=0.001)$. The level of NSE korelyuva in $(p<0,001)$ with the level of disability in called ' connection with a significant increase in blood at the blood-brain damaged bar ' EMU [21].Thus, there is currently no detailed information on the importance of NSE in cerebrovascular disease. P Rothe, because NSE is neyron- specific marker, it can be used ground to Define spare the degree of ischemic damage to the brain. The addition of scientific data on the influence of molecular pathogenetic factors on the course of the acute period of stroke was the subject of our study.

The aim of the study was to investigate the level of neuronspecific enolase in the acute period of ischemic stroke.

\section{Material and Research Methods}

We examined 85 patients (71 men and 14 women) with acute ischemic stroke (group 1), who were treated in the neuroreanimation and angioneurological departments of the Military Medical Clinical Center of the Western region (Lviv). The age of patients ranged from 31 to 87 years (mean age 61, $4 \pm 8$, 2 years). $31(36.5 \%)$ patients were diagnosed with a stroke in the left middle cerebral artery (LSMA) , 30 (35.3\%) - in the right middle cerebral artery (PSMA), 24 (28.2\%) - vertebrobasilar basin (VBB).The comparison group (group 2) consisted of 66 patients (52 men and 14 women) with chronic cerebral ischemia (CHI). The average age of patients was $61.6 \pm 8,5$ years. Among those surveyed with CHIM I stage were 20 people, X I M II stage - 46.As endpoint analyzed cases of stroke with fatal consequence that hanging up a Xia within 365 days after the second, whereby patients were roses in divisible into two groups - 73 patients and, who survived and 12 patients died, and during this period.

Clinical and diagnosis and II and CHEM established based on anamnesis, evaluation of subjective and objective neurological symptoms and the results of additional methods of examination (duplex scanning of the main arteries of the head, brain CT) according to ICD-10.Criteria for exclusion of patients from the study: cubarachnoid hemorrhage, cerebral hematoma , and other (non- 
vascular) diseases of the central nervous system, autoimmune, cancer, severe coronary heart disease , acute myocardial infarction , chronic pulmonary, renal or hepatic failure in stage diabetes mellitus, HIV infection.Evaluation of neurological status was standardized stroke scale for the National Institutes of Health (NIHSS): 0 points - no symptoms, 3 - 8 scores - easy neurologic deficits, 9-12 points - neurological deficit secondary hard spine 1315 points - severe neurological deficit, 16 - 34 points - extremely severe deficit, more than 34 points - coma $[2,4]$. Estimates of the level of consumer activity was evaluated and a scale daily nd of life Bartel well.

The maximum amount of points that response aye full of first independence in on vsyakdennomu life is 100 . Assessment of functional status was performed using a modified Rankin scalea. All patients performed neuropsychological examination using for orotk first scales and assessment of mental status (MMSE) [25].The level of NCE in all patients was determined by enzymelinked immunosorbent assay. In interpreting the results, the data of Hoffinian la Roche on the limits of normal values were taken into account. From these materials it follows that in $95 \%$ of serum samples from healthy people, the NCE values are below $15 \mathrm{ng} / \mathrm{ml}$ [26]. Blood for analysis of the level of NSE was taken on the day of admission - within 5 to 12 hours from the time of the onset of focal symptoms.In used application packages Statistica for Windows v. 8.0 (StatSoft Inc, USA, 2012) in accordance with the recommendations for processing the results of biomedical research.

\section{Results and Discussion}

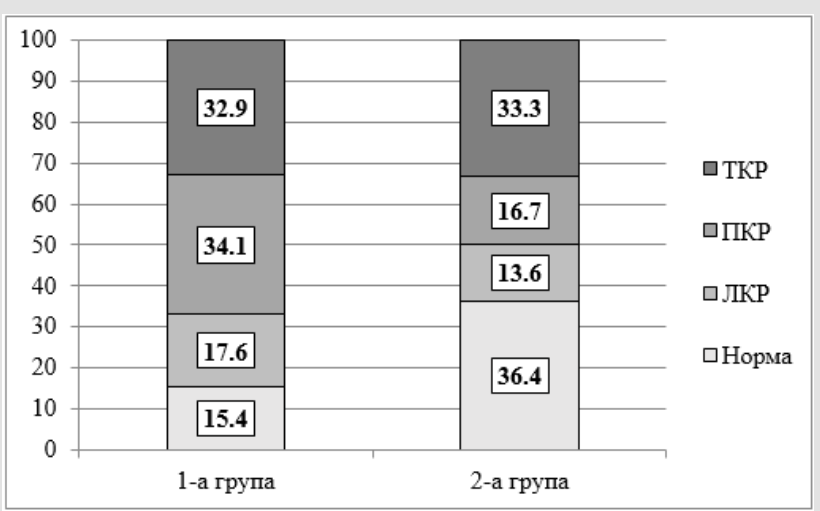

Figure 1: Distribution of patients (\%) of the studied groups according to the degree of cognitive disorders.

According to the NIHSS scale, the degree of neurological deficit in patients of the $1^{\text {st }}$ group ranged from 1 to 16 points (mean score was $10,7 \pm 0,6$ ). According to the severity of neurological disorders at the time of hospitalization, patients were divided as follows: 5 0 ( $58.8 \%$ ) - with a mild degree of neurological disorders $(5,9 \pm$ 0, 3 points); 27 ( $31.8 \%$ ) - with a neurological deficit of moderate severity $(10,8 \pm 0,7$ points); $8(9,4 \%)$ - with severe neurological deficit $(15,4 \pm 1,4$ points). The activity level on a scale Bartel and patients of group 1 ranged from 0 to 100 points, the average score was $65,8 \pm 10,2(p=0.049)$; in patients of the 2 nd group - $89.3 \pm 6$, 1 . The degree of disability for Rankin scale and ranged from 0 to 5 points, the average score was $3,3 \pm 0,8(p=0.02)$; patients in group $2-1,2 \pm 0$, 4.Upon admission, indicators of cognitive functions ranged from normal to severe disorders: the mean score on the MMSE scale in patients of group 1 was $24,3 \pm 0,5(p=0,046)$; patients in group $2-26,2 \pm 0,8$. Data on the distribution of patients according to the degree of cognitive disorders (CR) are presented in Figure 1.

Significant correlations of neurological deficit according to NIHSS with indicators of neuropsychological research according to MMSE ( $r=-0.48, p=0.001$ ) were established.In the acute stage of ischemic stroke avg is the value of serum NSE amounted to about $46,2 \pm 8,7 \mathrm{ng} / \mathrm{ml}$, which exceeded the value of similar th rate as patients in group 2 at 3,8 times $(\Delta \%=73.6 ; \mathrm{p}<0.001)$. In patients with CHM, the mean level of NCE was $12.2 \pm 2.3 \mathrm{ng} / \mathrm{ml}$. In stage I, CHEM average NSE no ref odyv beyond the maximum allowable concentrations of NSE according to the literature and was $10.1 \pm 1$ , $6 \mathrm{ng} / \mathrm{ml}$. For stage II characterized by a sharp increase of NSE 1.7 times $(17.1 \pm 3,1 \mathrm{ng} / \mathrm{ml})$ for equations in terms of stage I ( $\mathrm{p}$ $=0,047$ ) and a fairly large scale of values- 11,83 to $35,15 \mathrm{ng} /$ $\mathrm{m}$ l. Increase of NSE at CHEM bear $t b$ of part of neurodegenerative processes in the brain substance.When analyzing the difference in the level of NCE depending on sex, no significant dependence in patients of both groups was found. The average level of NCE in men was 42, $2 \pm 8,1 \mathrm{ng} / \mathrm{ml}$, in women $-50,2 \pm 9,8 \mathrm{ng} / \mathrm{ml} \mathrm{(} \mathrm{p}=0,530$ ). He Receive ly correlations th of NSE and age ( $r=0,174, p=0$, 415).

Provo ly pores and vnyannya of NSE in the onset of stroke localized lesions. The average level of NSE in the localization of the focus in the PSMA pool was 49, $3 \pm 9,1 \mathrm{ng} / \mathrm{ml}$, in the LSMA pool - 58, $2 \pm 10$, 8ng / ml, in VBB - 31, $1 \pm 7,4 \mathrm{ng} / \mathrm{ml}$. Significant differences were found in the level of NSE depending on the location of the ischemia - in the basin of LSMA and VBB ( $p=0,04)$. Differences between the localization of myocardial pool PSMA and LSMA ( $p=$ 0529) and PSMA and VBB $(p=0,123)$ were not.In the group of patients with severe neurologic deficit level of emergency $E$ was 63 , $8 \pm 11,3 \mathrm{ng} / \mathrm{ml}$, exceeding the indicator in patients with medium and light neurological deficit at $1,3(\Delta \%=25,9 ; \mathrm{p}=0,251)$ and $2,3(\Delta \%=56,9 ; p=0,007)$ times, respectively. The average level of NSE in patients with mild neurological deficit was 27, 5 $\pm 7,2 \mathrm{ng}$ / $\mathrm{ml}$ in AC andis NT and in with an average DEF and tsytom - 47, 3 $\pm 8,8 \mathrm{ng} / \mathrm{ml}$ (Figure 2). Trusted and $\mathrm{pH}$ and $\mathrm{X}$ differences he and vnya NSE between qi we group does not have to lo $(p=0,084)$. Established or direct strong correlation between the level of NCE and the severity of neurological deficit ( $r=0,67, p=0,027$ ), which indicates a direct relationship between the degree of ischemic injury and neurological disorders. The level of functional activity on the Barthel scale was significantly correlated with the level of NCE $(r=-0,58, p=0,032)$. 


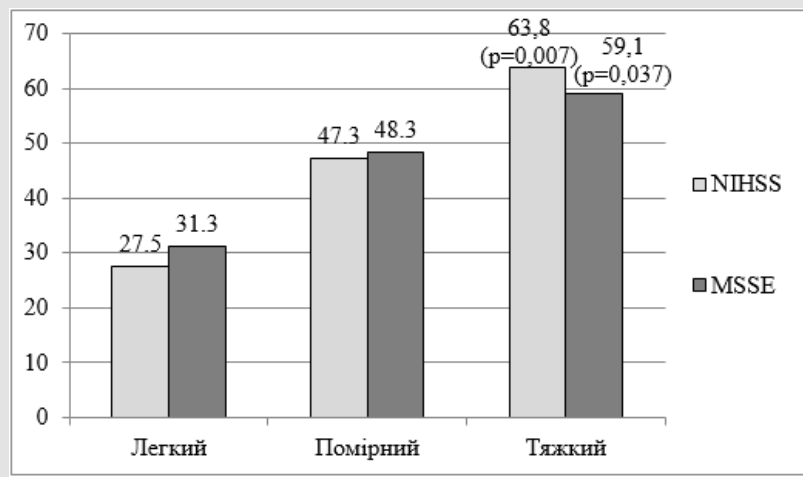

Figure 2: The level of NA E depending on the degree of neurological and cognitive deficits.

Note: Differently reliable between patients with severe and mild disorders.

A strong direct correlation of NSE with the level of disability on the Rankine scale ( $r=0,49, p=0,038$ ) was obtained.Thus, NCE directly reflects the depth and intensity of structural and functional disorders of biomembranes in the CNS, the severity of pathomorphological changes in neurons and the degree of permeability of the blood-brain barrier $[13,21]$. The obtained results testify to the informativeness of NCE in assessing the severity of patients in the acute period of ischemic stroke and justify the feasibility of including the detection of serum levels of NCE in routine clinical practice to optimize diagnostic measures in this group of patients.In the group of patients with TCR, the level of HCE was 59, $1 \pm 10,9 \mathrm{ng} / \mathrm{ml}$, which exceeded the value of patients with RCC and LCR in $1,2(\Delta \%=18,3 ; p=0,444)$ and in $1,9(\Delta \%$ $=47 ; p=0,037$ ) times respectively. The average level of NCE in patients with LCR was $31,3 \pm 7,5 \mathrm{ng} / \mathrm{ml}$, in patients with RCC 48, $3 \pm 8,9 \mathrm{ng} / \mathrm{ml}$ (Figure 2). Significant differences between NSE level qi we group would not in Lot $(p=0,146)$. A strong inverse correlation was found between the level of serum NS E and the MMSE score $(r=-0.73, p=0.037)$. The data with exclusion Insert about the possibility of determining the serum concentration of NAE for diagnostic validation rise manifestations Mr east stroke KP and Development Mr east stroke dementia.

When assessing the relationship between the level of NCE with the consequences of stroke (patients who survived and patients who died) revealed a tendency to higher NCE in patients who died, but this relationship did not reach the degree of reliability $(\mathrm{p}=$ 0,083 ). In patients who died level of NSE was $57,7 \pm 10,5 \mathrm{ng} /$ $\mathrm{ml}$ in patients who have you lived - 34, $8 \pm 7,8 \mathrm{ng} / \mathrm{ml}$.Identified threshold value Eq I NSE in the blood of patients with ischemic stroke, which corresponds to $40 \mathrm{ng} / \mathrm{ml}$. In patients with lethal outcome level NSE $\geq 40 \mathrm{ng} / \mathrm{ml}$ inst second in 8 (66.7\%) patients, patients and with satisfactory result - in $20(27,4 \%)$ patients $(\mathrm{p}=$ 0,028 ). Survival for a year in patients with levels of NSE $\leq 40 \mathrm{ng} /$
mL was 0.842 (95\% CI: [ $0.763-0.947$ ] , p <0,001), with a higher content of NSE - 0.495 (95\% CI: [0, $308-0.792]$, p <0.001).The presented data indicate that the level of NCE $\geq 40 \mathrm{ng} / \mathrm{ml}$ in the acute period of ischemic stroke is an independent risk factor for the development of fatal outcome during the year of observation. Thus, NSE has a high diagnostic value in the acute period of ischemic stroke and allows not only to assess the degree of brain tissue damage, but also allows to predict the course and possible consequences of ischemic stroke in the long term.

\section{Conclusion}

a) Determination of the brain damage marker - neuro $n$ spetsyf and including Noah enolaz and (NSE) in the plasma confirms the extent of damage to neurons and informative indicator of presence of neurological deficits in patients with acute ischemic stroke.

b) The value of the serum level of NSE in the onset of ischemic stroke correlates with the severity of neurological deficit on the NIHSS stroke scale ( $r=0.67, p=0.027$ ), with the level of functional activity on the Barthel scale $(r=-0.58, p=0.032)$, with the level of disability on the Rankine scale $(r=0.49, p=$ 0.038), with the severity of cognitive disorders on the MMSE test $(r=-0.73 ; p=0.037)$.

c) The results indicate a potential role definition NSE in risk stratification fatal consequences of in patients with ischemic stroke. Increase NSE $\geq 40 \mathrm{ng} / \mathrm{l}$ is predictive of unfavorable sign for shkodzhennyamo with fur.

d) Determination of NSE in blood serum should be included in a comprehensive examination of patients with acute ischemic stroke as a leading maker of cerebral injury.

\section{References}

1. Hankey GJ (2017) Stroke. Lancet 389(10069): 641-654.

2. Suslina ZA, Gulevskaya TS, Maksimova M Yu, Morgunov VA (2016) Cerebrovascular disorders: diagnosis, treatment, prevention. M: MED press-inform, pp. 536.

3. Yarosh AS, Pirogova LA, Filina NA (2014) The current state of the problem of acute cerebrovascular disorders . Journal Grodno State Medical University 3: 17-20.

4. Suslina ZA, Varakin Yu Ya (2009) Clinical and epidemiological research is a promising area of study of cerebral pathology (first report). Annals of clinical and experimental neurology 3(3): 4-11.

5. Lopez AD, Mathers CD, Ezzati M, Jamison DT, Murray CJL (2006) Global and regional burden of disease and risk factors, 2001: Systematic analysis of population health data. Lancet 367(9524): 1747-1757.

6. Syskina EN (2011) Biochemical markers of brain tissue condition and hemostasis in patients with severe cerebral infarction in the arteries of the carotid system: author's ref. dis. ... Cand. honey. Science. 14.01.11 "nervous diseases", 03.01 .04 - "biochemistry". M, p. 24.

7. Shabalina AA (2008) Hemostasis and biochemical markers of brain tissue damage in atherothrombotic and lacunar subtypes of ischemic stroke: author's ref. dis. ... Cand. honey. Science. 14.00.13 - "N eral diseases ", 03.00 .04 - "Biological chemistry ". M, p. 31. 
8. Koh PO (2010) Proteomic analysis of focal cerebral ischemic injury in male rats . J. Vet. Med. Sci 72(2): 181-185.

9. Whiteley W, Tseng MC, Sandercock P (2008) Blood biomarkers in the diagnosis of ischemic stroke. A sys-thematic review. Stroke 39(10): 2902-2909.

10. Jauch EC, Lindsell C, Broderick J, Fagan SC, Tilley BC, et al. (2006) Association of serial biochemical markers with acute ischemic stroke. The National Institute of Neurological Disorders and Stroke recombinant tissue plasminogen activator stroke study . Stroke 37(10): 2508-2513.

11. Butterworth RJ, Wassif WS, Sherwood RA, Gerges A, Poyser KH, et al. (1996) Serum neuron-specific enolase, carnosinase, and their ratio in acute stroke. An enzymatic test for predicting outcome? Stroke 27(11): 2064-2068.

12. Zierath D, Thullbery M, Hadwin J, Gee JM, Savos A, et al. (2010) CNS immune responses fo llowing experimental stroke. Neurocrit Care 12(2): $274-284$.

13. Pandey A, Saxena K, Verma M, Bharosay A (2011) Correlative study between neuron-specific enolase and blood sugar level in ischemic stroke patients. J Neurosci Rural Pract 2(1): 50-54.

14. Nayak AR, Badar SR, Lande N, Kawle AP, Kabra DP, et al. (2016) Prediction of outcome in diabetic acute ischemic stroke patients: a hospital-based pilot study report. Ann Neurosci 23(4): 199-208.

15. Rodrigues Yaňez M, Castellanos M, Sobrino T, Brea D, Ramos Cabrer P, et al. (2013) Interleukin-10 facilitates the selection of patients for systemic thrombolysis . BMC Neurol 13: 62.

16. Jung CS, Lange B, Zimmermann M, Seifert V (2013) CSF and serum biomarkers focusing on cerebral vasospasm and ischemia after subarachnoid hemorrhage. Stroke research and treatment 2013: 560305.

17. Alatas ÖD, Gürger M, Atesscelik M, Yildiz M, Demir CF, et al. (2015) Neuron-specific enolase, S100 calcium-binding protein B, and heat shock protein 70 levels in patients with intracranial hemorrhage. Medicine 94(45): e2007.

\section{ISSN: 2574-1241}

DOI: $10.26717 /$ BJSTR.2020.31.005030

Saiko OV. Biomed J Sci \& Tech Res

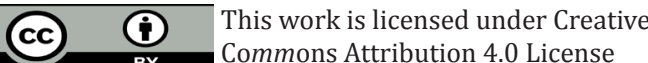

Submission Link: https://biomedres.us/submit-manuscript.php
18. Dementieva OV, Starikova NL (2017) Clinical correlations of neuronspecific enolase in patients with lifelong ischemic stroke after systemic thrombolysis. Ural Medical Journal 1(145): 32-36.

19. Browns R, De Will B, Cras P, Surgeloose DD, Mariën P, et al. (2010) Neurobiochemical markers of brain damage in cerebrospinal fluid of acute ischemic stroke patients . Clinical Chemistry 56(3): 451-458.

20. Anand N, Stead LG (2005) Neuron-specific enolase as a marker for acute ischemic stroke: a systematic review. Cerebrovasc Dis 20(4): 213-219.

21. Bharosay A, Bharosay VV, Varma M, Saxena K, Sodani A, et al. (2012) Correlation of brain biomarker neuron specific enolase (NSE) with degree of disability and neurological worsening in cerebrovascular stroke. Ind J Clin Biochem 27(2): 186-190.

22. Kawle AP, Nayak AR, Lande NH, Kabra DP, Chandak NH, et al. (2015) Comparative evaluation of risk factors, outcome and biomarkers levels in young and old acute ischemic stroke patients. Annals of Neurosciences 22(2): 70-77.

23. Zaheer S, Beg M, Rizvi I, Islam N, Ullah E, et al. (2013) Correlation between serum neuron specific enolase and functional neurological outcome in patients of acute ischemic stroke . Ann Indian Acad Neurol 16(4): 504-508.

24. Gonzalez Garcia S, Gonzalez Quevedo A, Fernandez O, Sánchez MP, Saínz CM, et al. (2012) Concepcion Short-term prognostic value of serum neuron specific enolase and S100B in acute stroke patients. $\mathrm{Cl}$ in Biochem 45(16-17): 1302-1307.

25. Belova AN (2004) Scales, tests and questionnaires in neurology and neurosurgery. A guide for doctors. Medkniga, Moscow, Russia, pp. 456.

26. Toropova NE, Dorofeeva EA, Dvoryaninova SP, Vasieva Zh P (1995) Comments on the information content of a neuron - a special enolase, determined by the enzyme immunoassay. Clinical laboratory e and agnostics 1: 15-17.

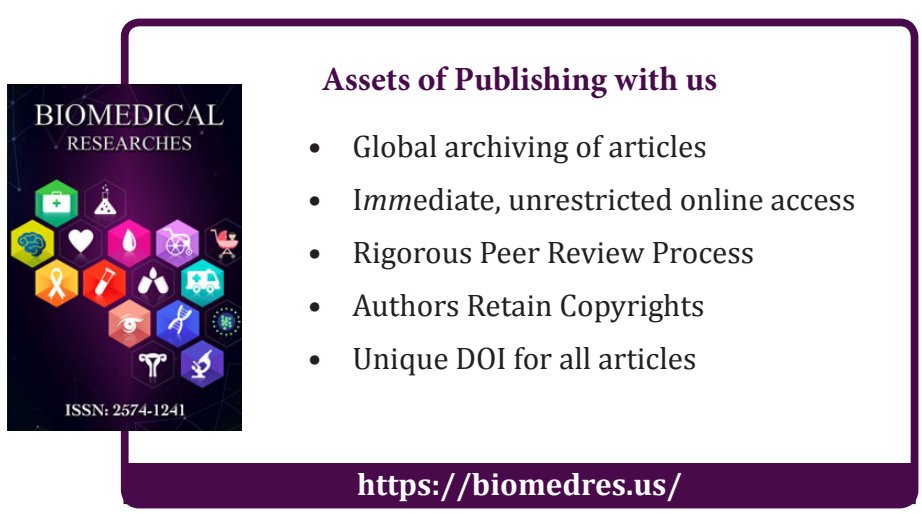

